\title{
The impact of mental fatigue on brain activity: a comparative study both in resting state and task state using EEG
}

\author{
Gang Li', Shan Huang ${ }^{1 *} \mathbb{0}$, Wanxiu Xu' ${ }^{1}$, Weidong Jiao ${ }^{1}$, Yonghua Jiang ${ }^{1}$, Zhao Gao ${ }^{1}$ and Jianhua Zhang ${ }^{2^{*}}$
}

\begin{abstract}
Background: Mental fatigue is usually caused by long-term cognitive activities, mainly manifested as drowsiness, difficulty in concentrating, decreased alertness, disordered thinking, slow reaction, lethargy, reduced work efficiency, error-prone and so on. Mental fatigue has become a widespread sub-health condition, and has a serious impact on the cognitive function of the brain. However, seldom studies investigate the differences of mental fatigue on electrophysiological activity both in resting state and task state at the same time. Here, twenty healthy male participants were recruited to do a consecutive mental arithmetic tasks for mental fatigue induction, and electroencephalogram (EEG) data were collected before and after each tasks. The power and relative power of five EEG rhythms both in resting state and task state were analyzed statistically.

Results: The results of brain topographies and statistical analysis indicated that mental arithmetic task can successfully induce mental fatigue in the enrolled subjects. The relative power index was more sensitive than the power index in response to mental fatigue, and the relative power for assessing mental fatigue was better in resting state than in task state. Furthermore, we found that it is of great physiological significance to divide alpha frequency band into alpha1 band and alpha 2 band in fatigue related studies, and at the same time improve the statistical differences of sub-bands.
\end{abstract}

Conclusions: Our current results suggested that the brain activity in mental fatigue state has great differences in resting state and task state, and it is imperative to select the appropriate state in EEG data acquisition and divide alpha band into alpha1 and alpha2 bands in mental fatigue related researches.

Keywords: Mental fatigue, EEG, Resting state, Task state

\section{Background}

Mental fatigue refers to a condition of low alertness and cognitive impairment [1]. Too much brain activity and stimulation can make a person feel mentally exhausted, and this feeling is akin to physical fatigue. Mental fatigue

\footnotetext{
*Correspondence: 641822416@qq.com; jhzhang@sdu.edu.cn

${ }^{1}$ College of Engineering, Zhejiang Normal University, 688 Yingbin Road,

Zhejiang 321004 Jinhua, People's Republic of China

${ }^{2}$ Key Laboratory of High Efficiency and Clean Mechanical Manufacture,

Ministry of Education of China, School of Mechanical Engineering,

Shandong University, 688 Yingbin Road, Zhejiang 321004 Jinan, People's

Republic of China
}

can give rise to numerous bad consequences, for example, making the uncomplicated tasks turn to be increasingly difficult or even impossible. Mental fatigue has become a popular sub-healthy state in nowadays, which has effects on almost all aspects of cognitive functions of human brain [2], such as driving fatigue [3]. Considering the impacts of mental fatigue on our daily life, it is very important to reveal the differences of mental fatigue on brain activity in resting state and task state at the same time.

Previous studies have been centered on the changes associated with task-related brain activity $[4,5]$. The 
fatigue-inducing mental tasks were widely used by researchers. The mental tasks that requiring different intensity of attention can differentiate the levels of mental fatigue, and their experimental duration could be distinct. The $n$-back task $[6,7]$ and psychomotor vigilance task $[8,9](\mathrm{PVT})$ can be categorized as high-attentiondemanding tasks, sleep deprivation [10] can be classified into low-attention-demanding tasks, meanwhile mental arithmetic task [11] and driving simulation task [12] fall into middle-attention-demanding tasks. However, among these three-type tasks, the middle-attention-demanding tasks are greatly in line with our daily working load. Therefore, we chose mental arithmetic task to induce mental fatigue.

Historically, mental fatigue has been most prevalently studied with the neuroimaging technique of EEG [1315]. It has been widely proved that mental fatigue can result in obvious changes in EEG signals [16]. Strijkstra has found that EEG at resting state shows strong negative correlations of alpha power and positive correlations of theta power with subjective sleepiness [17]. With the mental fatigue increasing, the power of alpha rhythm increases when the eyes are open and decreases when the eyes are closed [18]. These changes in EEG can be used to detect mental fatigue $[11,16]$, which is especially important and meaningful for driving fatigue estimation $[8,12]$. From the above, we can conclude that EEG has become the most effective technical means for exploring the neuromechanism and detection of mental fatigue $[19,20]$.

The current study is also motivated by the studies which have divided the EEG bands into narrower bands. In mental fatigue related studies, some researchers divided alpha band into alpha1 $(8-10 \mathrm{~Hz})$ and alpha2 $(10-13 \mathrm{~Hz})$. Li has performed statistical analysis on the characteristics of alpha1 and alpha2 to estimate mental fatigue, and reported that alpha1 band is better for fatigue detection [11]. Sun has applied alpha1 frequency band for mental fatigue classification, and achieved a high prediction accuracy [21].

In this study, we would explore the differences of mental fatigue on electrophysiological activity both in resting and task states at the same time. To this end, we administered a group of challenging sustained mental arithmetic math tasks for mental fatigue induction among the recruited young healthy male participants, and EEG data for resting and task states before and after each task segments were collected. Then the power and relative power of delta, theta, alpha1, alpha2, and beta were computed, and statistical analysis were carried on the results among different brain regions.

\section{Results}

Figure 1 and Table 1 depict the results of EEG power. It has been found that the power of each rhythm in resting state and task state varies in the evolutionary process of mental fatigue, but there are few statistical differences and FDR correction are not performed on the results of the $P$-values. In resting state, the alphal rhythm power at frontal and temporal regions have a marked tesndency to increase, and the alpha2 power at parietal region has a significant reduction. In task state, only the beta power at parietal and occipital regions have obvious decrease. The reason for the insignificant statistical differences may be that the power of EEG rhythms is not sensitive to the mental fatigue, and the small changes of EEG power will be masked by individual differences and power spectrum fluctuations. Therefore, the other widely used EEG index, relative power, was analyzed in the study.

Figures 2 and 3, Tables 2, and 3 show the results of EEG relative power in resting state. Figure 2 is the brain topography of the relative power for every rhythms. Figure 3 is the average relative power of all electrodes.
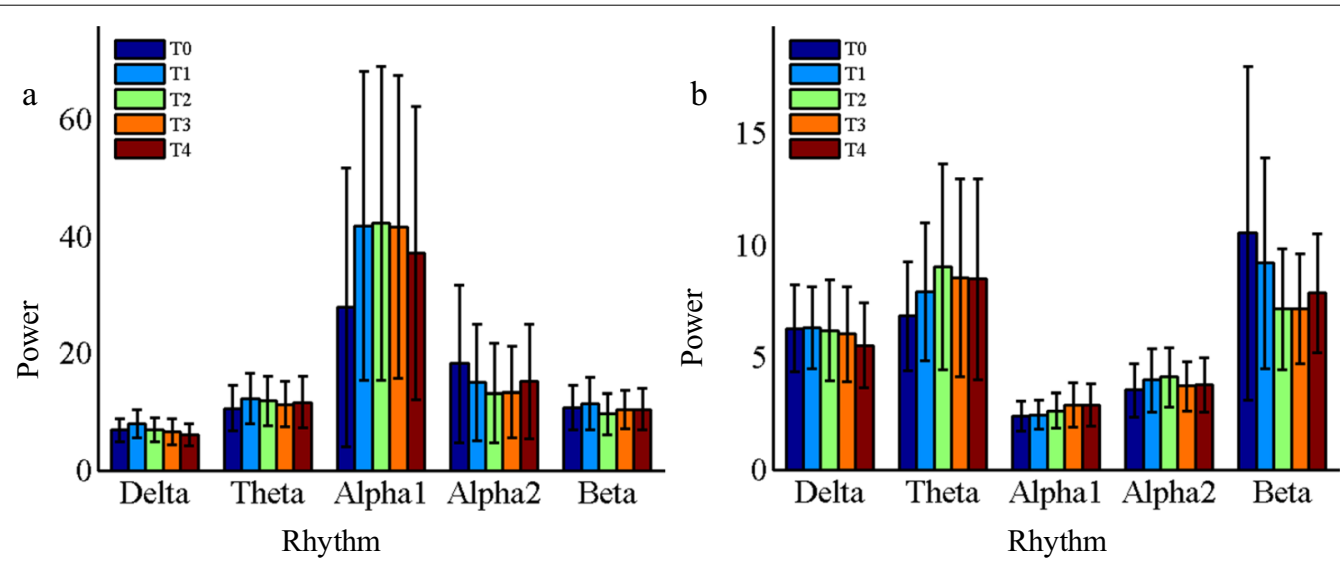

Fig. 1 The power of EEG rhythms over the whole brain region at different time periods. a Resting state, $\mathbf{b}$ Task state 
Table 1 ANOVA results of $P$-value for EEG power in different brain regions in resting state and task state

\begin{tabular}{|c|c|c|c|c|c|c|}
\hline State & Region & Delta & Theta & Alpha1 & Alpha2 & Beta \\
\hline \multirow[t]{6}{*}{ Resting state } & Whole & 0.118 & 0.792 & 0.403 & 0.572 & 0.710 \\
\hline & Frontal & 0.366 & 0.819 & 0.044 & 0.372 & 0.656 \\
\hline & Temporal & 0.135 & 0.574 & 0.011 & 0.221 & 0.078 \\
\hline & Central & 0.513 & 0.887 & 0.119 & 0.114 & 0.273 \\
\hline & Parietal & 0.141 & 0.735 & 0.203 & $4.3 E-4$ & 0.112 \\
\hline & Occipital & 0.492 & 0.796 & 0.914 & 0.159 & 0.089 \\
\hline \multirow[t]{6}{*}{ Task state } & Whole & 0.760 & 0.507 & 0.212 & 0.673 & 0.103 \\
\hline & Frontal & 0.833 & 0.728 & 0.735 & 0.390 & 0.684 \\
\hline & Temporal & 0.106 & 0.179 & 0.765 & 0.903 & 0.193 \\
\hline & Central & 0.965 & 0.845 & 0.434 & 0.743 & 0.195 \\
\hline & Parietal & 0.644 & 0.426 & 0.059 & 0.057 & $8.9 E-7$ \\
\hline & Occipital & 0.223 & 0.724 & 0.757 & 0.324 & 0.013 \\
\hline
\end{tabular}
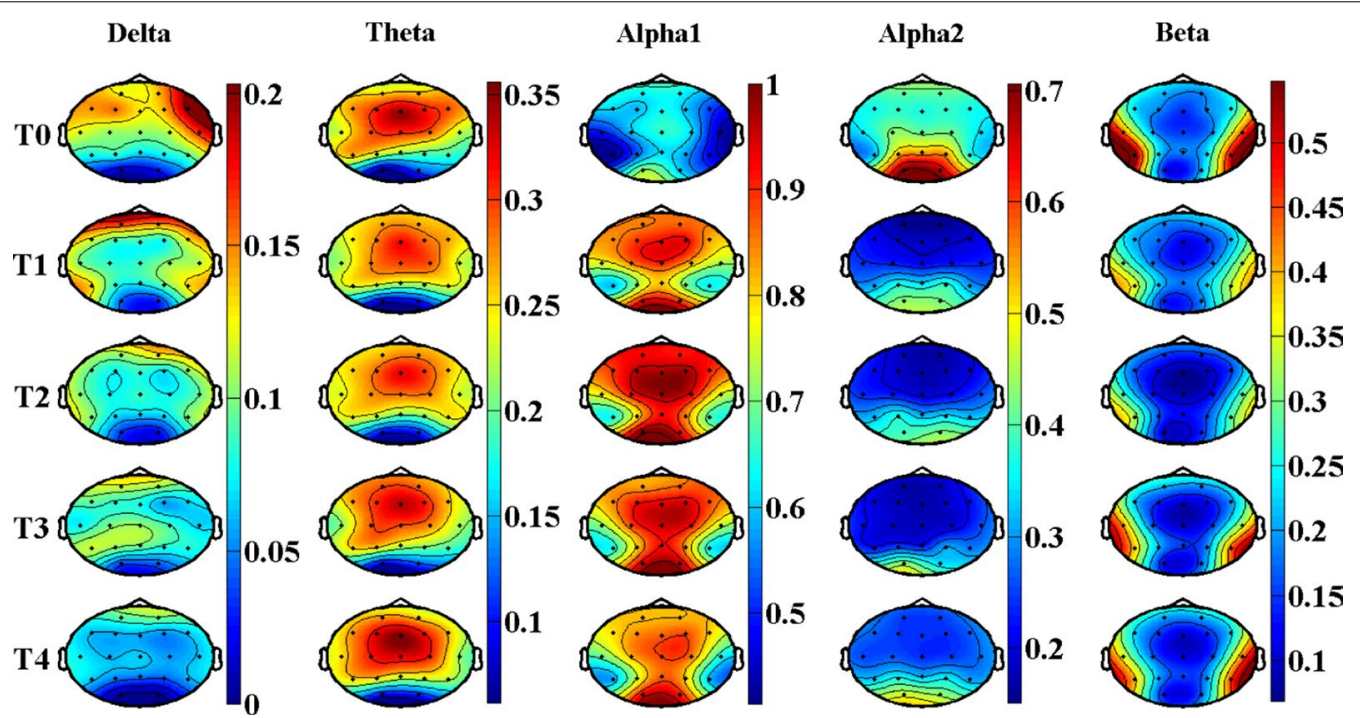

Fig. 2 Brain topography of the relative power of EEG rhythms in resting state. In the figure, all the relative power values are normalized to 0-1

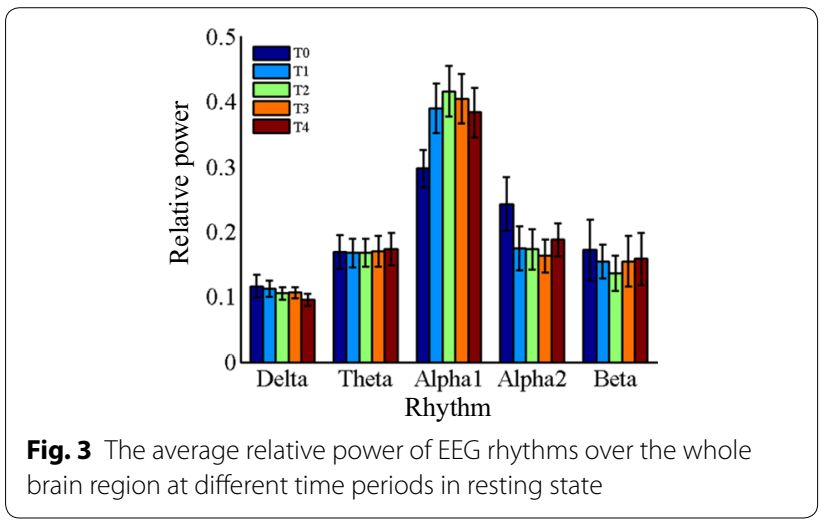

Table 2 is the statistical results of $P$ value, $F$-value and $\eta_{p}^{2}$ for EEG relative power in different brain regions, which shows very good statistical differences according to the results of $P$-value, $F$-value and $\eta_{p}^{2}$. Therefore, FDR correction was performed on the results of $P$-value among different brain regions to reduce the risk of false positive. Table 3 is the corrected statistical differences corresponding to Table 2. As shown in Figs. 2 and 3, Tables 2 and 3, the relative power of delta rhythm presents a monotonically decreasing trend throughout the whole brain, and has corrected significant statistical differences across all brain regions. The results of theta rhythm indicate that only in the central region has a corrected significant increasing trend 
Table 2 ANOVA results of $P$-value, $F$-value and $\eta_{p}^{2}$ for relative power EEG rhythms in different brain regions in resting state

\begin{tabular}{|c|c|c|c|c|c|c|c|c|c|c|c|c|c|c|c|}
\hline \multirow[t]{2}{*}{ Region } & \multicolumn{3}{|l|}{ Delta } & \multicolumn{3}{|l|}{ Theta } & \multicolumn{3}{|l|}{ Alpha1 } & \multicolumn{3}{|l|}{ Alpha2 } & \multicolumn{3}{|l|}{ Beta } \\
\hline & $P$ & $F$ & $\eta_{p}^{2}$ & $P$ & $F$ & $\eta_{p}^{2}$ & $P$ & $F$ & $\eta_{p}^{2}$ & $P$ & $F$ & $\eta_{p}^{2}$ & $P$ & $F$ & $\eta_{p}^{2}$ \\
\hline Whole & $1.5 E-5$ & 7.98 & 0.26 & 0.955 & 0.17 & 0.01 & $1.4 E-15$ & 29.3 & 0.57 & $7.1 E-11$ & 18.0 & 0.44 & 0.079 & 2.16 & 0.09 \\
\hline Frontal & $2.2 E-5$ & 10.3 & 0.58 & 0.664 & 0.60 & 0.07 & $3.4 E-15$ & 75.2 & 0.91 & $4.5 E-20$ & 168 & 0.96 & 0.038 & 2.91 & 0.28 \\
\hline Temporal & 0.025 & 3.81 & 0.50 & 0.727 & 0.51 & 0.12 & $2.3 E-4$ & 10.9 & 0.74 & 0.012 & 4.72 & 0.56 & $7.3 E-5$ & 13.5 & 0.78 \\
\hline Central & $2.0 E-4$ & 16.5 & 0.87 & 0.005 & 7.29 & 0.74 & 1.7E-6 & 47.9 & 0.95 & $2.1 E-9$ & 193 & 0.99 & 0.117 & 2.42 & 0.09 \\
\hline Parietal & $1.8 E-4$ & 17.1 & 0.86 & 0.309 & 1.38 & 0.06 & $5.5 E-4$ & 13.1 & 0.84 & $1.2 E-5$ & 31.7 & 0.93 & 0.043 & 3.68 & 0.59 \\
\hline Occipital & 0.015 & 9.42 & 0.89 & 0.950 & 0.16 & 0.03 & 0.004 & 16.4 & 0.93 & 0.002 & 22.3 & 0.95 & 0.750 & 0.48 & 0.02 \\
\hline
\end{tabular}

Statistically significant differences are highlighted in italics $(P<0.05)$

Table 3 Corrected results of $P$-value corresponding to Table 2 among different brain

\begin{tabular}{llllll}
\hline Region & Delta & Theta & Alpha1 & Alpha2 & Beta \\
\hline Whole & $6.6 E-5$ & 0.955 & $8.4 E-15$ & $2.1 E-10$ & 0.1185 \\
Frontal & $6.6 E-5$ & 0.955 & $1.0 E-14$ & $2.7 E-19$ & 0.086 \\
Temporal & 0.025 & 0.955 & $3.5 E-5$ & 0.012 & $4.4 E-4$ \\
Central & $3.0 E-4$ & 0.030 & $3.4 E-6$ & $4.2 E-9$ & 0.1404 \\
Parietal & $3.0 E-4$ & 0.927 & $6.6 E-4$ & $1.8 E-5$ & 0.086 \\
Occipital & 0.018 & 0.955 & 0.004 & $2.4 E-3$ & 0.75
\end{tabular}

Statistically significant differences are highlighted in italics $(P<0.05)$

$\left[P=0.005, F=7.29, \eta_{p}^{2}=0.74\right.$, corrected $\left.P=0.03\right]$, and there are no statistic difference in other brain regions. Whereas, both alpha1 rhythm and alpha2 rhythm have corrected significant statistical differences among all the brain regions, and beta rhythm have corrected significant statistical differences only in temporal region $\left[P=7.3 \mathrm{E}-5, \quad F=13.5, \quad \eta_{p}^{2}=0.78\right.$, corrected $P=4.4 \mathrm{E}-4]$. Besides, the changing regularities of alpha1, alpha2, and beta rhythms are not monotonous.

Figures 4 and 5, Tables 4 and 5 show the results of EEG relative power in task state. Figure 4 is the brain topography of the relative power for every rhythms, and Fig. 5 is the average relative power of all electrodes. Table 4 is the statistical results of $P$-value, $F$-value and $\eta_{p}^{2}$ for EEG relative power in different brain regions. Table 5 is the corrected statistical differences corresponding to Table 4 obtained by FDR correction performed on the results of $P$-value among different brain regions. As shown in Figs. 4 and 5, Tables 4 and 5, the relative power of delta rhythm presents a decreasing trend throughout the whole brain, and has corrected significant statistical differences in frontal region $\left[P=0.003, F=5.22, \eta_{p}^{2}=0.41\right.$, corrected $P=0.012]$, central region $[P=0.004, F=7.69$, $\eta_{p}^{2}=0.76$, corrected $\left.P=0.012\right]$, and parietal region

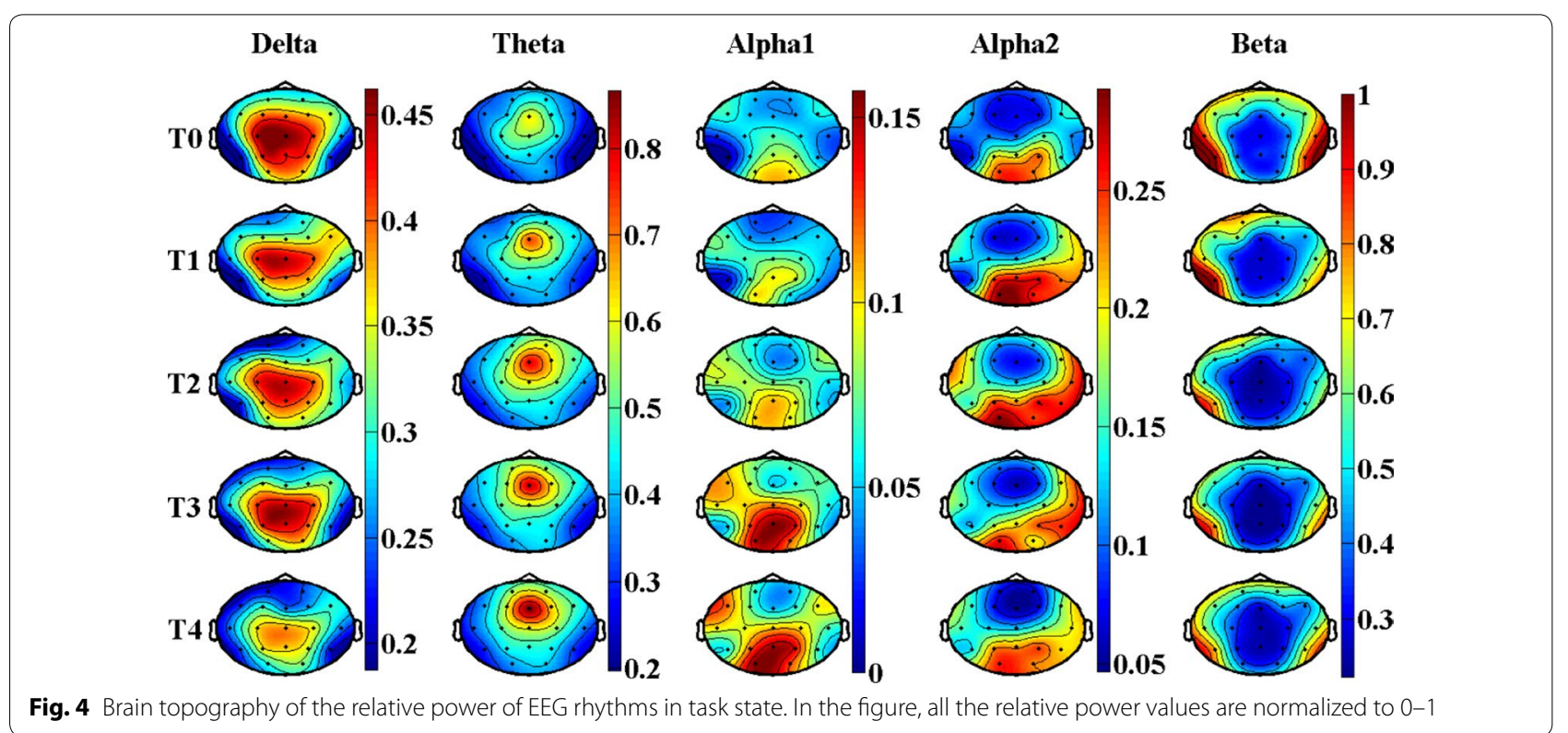




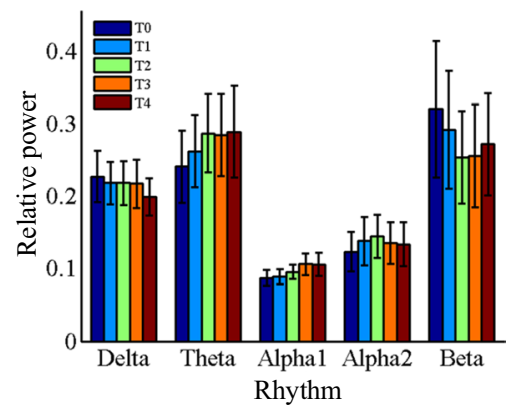

Fig. 5 The average relative power of EEG rhythms over the whole brain region at different time periods in task state

$\left[P=0.006, F=7.02, \eta_{p}^{2}=0.74\right.$, corrected $\left.P=0.012\right]$. Both theta rhythm and alphal rhythm have a non-monotonic increasing trend for the results of relative power, but only in temporal region for theta rhythm $[P=0.001, F=7.67$, $\eta_{p}^{2}=0.67$, corrected $\left.P=0.006\right]$, and only in central region $\left[P=7.7 \mathrm{E}-5, F=20.8, \eta_{p}^{2}=0.89\right.$, corrected $\left.P=4.6 \mathrm{E}-4\right]$ and parietal region $\left[P=3.6 \mathrm{E}-4, F=14.5, \eta_{p}^{2}=0.85\right.$, corrected $P=0.001]$ for alpha1 rhythm that have corrected significant statistical differences. As for alpha2 rhythm and beta rhythm, no corrected statistically significant differences are observed.

\section{Discussion}

In the present study, we analyzed the difference in spontaneous neural activities caused by performing prolonged fatigue-inducing mental arithmetic tasks both in resting state and task state. Five EEG rhythms were evaluated among five brain regions in the two states. The delta rhythm power was $7.1 \pm 0.54 \mu \mathrm{V}^{2}$ in the resting state, and had the lowest proportion (10\%) in all EEG rhythms; in the task state, the power was $6.1 \pm 0.34 \mu \mathrm{V}^{2}$, but the proportion increased to $21.5 \%$. This is mainly because Alpha1 and Alpha2 rhythms were significantly suppressed in task state (see Fig. 4), leading to a significant increase in the proportion of corresponding delta rhythm. The delta rhythm power had no statistical difference both in resting
Table 5 Corrected results of $P$-value corresponding to Table 4 among different brain

\begin{tabular}{llllll}
\hline Region & Delta & Theta & Alpha1 & Alpha2 & Beta \\
\hline Whole & 0.131 & 0.088 & 0.112 & 0.622 & 0.180 \\
Frontal & 0.012 & 0.171 & 0.138 & 0.648 & 0.286 \\
Temporal & 0.406 & 0.006 & 0.225 & 0.492 & 0.090 \\
Central & 0.012 & 0.509 & $4.6 E-4$ & 0.859 & 0.326 \\
Parietal & 0.012 & 0.063 & 0.001 & 0.492 & 0.206 \\
Occipital & 0.056 & 0.579 & 0.114 & 0.648 & 0.286 \\
\hline
\end{tabular}

Statistically significant differences are highlighted in italics $(P<0.05)$

and task states (see Table 6), which is in line with its actual physiological meaning. Because delta rhythm is related to people's deep sleep [22], and it usually appears in large quantities in adult's deep sleep, anesthesia and hypoxia. As for the relative power of delta rhythm, it decreased significantly along with the accumulation of task time, which is consistent with the results reported by Jap when researching driving fatigue [23]. Some literatures have also pointed out that the amplitude and relative power of delta rhythm increased under fatigue state [24]. However, in many fatigue evaluation studies, delta band was directly removed by researchers and technicians [22]. Because they believe that delta rhythm reflects the state of deep sleep, and general brain fatigue status does not show significant changes. Moreover, the frequencies of EEG artifacts (such as blink artifacts, eye movement artifacts, electrocardio artifacts, etc., except for power-frequency artifacts and myoelectricity artifacts) mainly coincide with the delta frequency band. The removal of the artifacts is highly subjective, and the removal effect varies from person to person. Therefore, the results of delta rhythm in this study will not be in further discussion.

The power and relative power results of theta rhythm were unanimous both in resting state and task state, demonstrating an increasing trend, which was consistent with the results of most fatigue studies [17, 22-24]. Generally, theta rhythm is considered to reflect the early state of sleepiness [25], which is related to brain fatigue [26]

Table 4 ANOVA results of $P$-value, $F$-value and $\eta_{p}^{2}$ for relative power EEG rhythms in different brain regions in task state

\begin{tabular}{|c|c|c|c|c|c|c|c|c|c|c|c|c|c|c|c|}
\hline \multirow[t]{2}{*}{ Region } & \multicolumn{3}{|l|}{ Delta } & \multicolumn{3}{|l|}{ Theta } & \multicolumn{3}{|l|}{ Alpha1 } & \multicolumn{3}{|c|}{ Alpha2 } & \multicolumn{3}{|l|}{ Beta } \\
\hline & $P$ & $F$ & $\eta_{p}^{2}$ & $P$ & $F$ & $\eta_{p}^{2}$ & & $F$ & $\eta_{p}^{2}$ & $P$ & $F$ & $\eta_{p}^{2}$ & $P$ & $F$ & $\eta_{p}^{2}$ \\
\hline Whole & 0.109 & 1.95 & 0.08 & 0.044 & 2.56 & 0.10 & 0.056 & 8.99 & 0.29 & 0.311 & 1.21 & 0.05 & 0.060 & 2.53 & 0.09 \\
\hline Frontal & 0.003 & 5.22 & 0.41 & 0.114 & 2.04 & 0.11 & 0.115 & 3.68 & 0.33 & 0.526 & 0.81 & 0.09 & 0.197 & 1.62 & 0.11 \\
\hline Temporal & 0.406 & 1.07 & 0.02 & 0.001 & 7.67 & 0.67 & 0.225 & 3.80 & 0.50 & 0.084 & 2.53 & 0.4 & 0.015 & 4.41 & 0.54 \\
\hline Central & 0.004 & 7.69 & 0.76 & 0.424 & 1.06 & 0.02 & $7.7 E-5$ & 20.8 & 0.89 & 0.859 & 0.32 & 0.01 & 0.326 & 1.32 & 0.05 \\
\hline Parietal & 0.006 & 7.02 & 0.74 & 0.021 & 4.72 & 0.65 & $3.6 E-4$ & 14.5 & 0.85 & 0.164 & 2.04 & 0.12 & 0.103 & 8.25 & 0.07 \\
\hline Occipital & 0.037 & 6.03 & 0.83 & 0.579 & 0.79 & 0.03 & 0.076 & 4.12 & 0.27 & 0.540 & 0.87 & 0.02 & 0.238 & 2.03 & 0.13 \\
\hline
\end{tabular}

Statistically significant differences are highlighted in italics $(P<0.05)$ 
Table 6 Division of the brain regions and their included electrode

\begin{tabular}{ll}
\hline Brain region & Electrode name \\
\hline Frontal & $\mathrm{Fp} 1, \mathrm{Fp} 2, \mathrm{~F} 3, \mathrm{~F} 4, \mathrm{~F} 7, \mathrm{~F} 8, \mathrm{Fz}$ \\
Temporal & $\mathrm{T} 3, \mathrm{~T} 4, \mathrm{~T} 5, \mathrm{~T} 6$ \\
Central & $\mathrm{C} 3, \mathrm{Cz}, \mathrm{C} 4$ \\
Parietal & $\mathrm{P} 3, \mathrm{Pz}, \mathrm{P} 4$ \\
Occipital & $\mathrm{O} 1, \mathrm{O} 2$ \\
\hline
\end{tabular}

and has a sensitive response to fatigue [27]. As shown in Tables 3 and 5, the response results of theta rhythm in the task state were slightly better than that in the resting state, because there were corrected statistical differences in the temporal region and parietal region in the task state, while there were corrected statistical differences only in the central region in the resting state.

Alpha rhythm reflects the state of relaxation and wakefulness. When focusing attention, external stimulation or visual input, alpha rhythm will be blocked [28]. Alpha rhythm is considered to be the most sensitive indicator of brain fatigue [26, 27], which is consistent with the statistical analysis results of alpha1 and alpha2 showed in Tables 2, 3, 4, and 5. Along with the increase of mental fatigue, the power and relative power of alpha rhythm were reported to be significantly increased [22, 24]. Several other researchers reported the opposite changing tendency [23]. However, it is now widely accepted that alpha rhythm intensifies as the brain transformed from normal into fatigue $[29,30]$ (see detailed statistical results of relevant studies in Ref. [27]). As shown in Tables 3 and 5, the effect of alpha1 and alpha2 rhythm in depicting mental fatigue in resting state is better than that in task state.

In this study, alpha band was further divided into two sub-bands, alpha1 band and alpha2 band, obtaining some meaningful results: the relative power of alpha1 rhythm increased significantly both in resting state and task state, while alpha 2 rhythm decreased significantly in the resting state, but showed an increasing trend in the task state, which were consistent with the power change trends shown in Fig. 1. In similar research results, it is also pointed out that alpha1 rhythm power increases with the increase of fatigue level [21, 30,31], and alpha 2 rhythm has the same changes in task state [30]. The changing regularities of alpha1 and alpha2 in the resting state is completely opposite, and that in the task state is consistent, indicating that it is essential to divide alpha frequency band into alpha 1 and alpha2 subbands in brain fatigue research based on EEG. Klimesch has emphasized that using narrower frequency bands in the study can reduce the risk that the frequency effects are cancelled out or not discovered [32], which is well demonstrated in the results of alpha1 and alpha2 rhythms in this study. In addition, narrower frequency band division can enhance the physiological meaning of the subbands and make their statistical results more significant. The contrary changing trend and significant statistical results of Alpha1 and Alpha2 rhythms in the resting state can prove this inference.

Klimesch has pointed out through the analysis of event-related potential that alpha1 rhythm is related to attention, and its power will increase significantly when the attention task increases and the subjects are required to stay awake and not allow sleep and rest [32], which is consistent with the results of alpha1 rhythm in this study. As for alpha2 rhythm, Klimesch et al. have indicated that alpha2 desynchronization is positively correlated with brain long-term memory function by comparing the performance of subjects with different memory abilities in memory tasks [33]. In their subsequent studies, it has been further proved that alpha2 rhythm is correlated with memory [34-37] and cognitive behavior [38]. When the memory task increases, alpha2 rhythm (in the state of eye closure at the time of EEG data collection) shows synchronization $[34,35,39]$, that is, the power decreases, which can explain the changing trend of alpha2 rhythm in the resting state in this study.

Further analysis of the brain topography in the fourth column of Figs. 2 and 4, we found that: (a) alpha2 rhythm power is mainly distributed in the occipital region, which is consistent with the results of topography given by Craig et al. [30]; (b) in resting state, alpha2 rhythm is very strong in all brain regions in the baseline state (referring to the T0 period), but when the brain enters into the fatigue state (referring to the T1, T2, T3 and T4 periods), alpha2 rhythm is mainly concentrated in the occipital region; (c) in task state, alpha2 rhythm is mainly concentrated in occipital region, and tends to strengthen in the parietal region and right temporal region with the increase of tasks. The above results suggest that alpha2 rhythm is also closely related to visual information processing in the brain, as the occipital lobe is mainly responsible for visual functions. In the resting state, there is no visual information input in the brain, and the influence of memory task may be dominant in the brain, so the brain is shown as de-synchronization [33], and the power and relative power are shown as decreased. In the task state, the brain has a large amount of visual information to be processed, then the neural centers in the occipital area and nearby brain areas are activated (manifested as increased energy of alpha2 rhythm) to complete the visual information transmission and processing tasks. The influence of visual information processing task is dominant, while the influence of memory task is covered. Under the combined action of these two comprehensive 
effects, the power of alpha2 rhythm has increasing trend, but no statistical difference.

With the deepening of mental fatigue, the relative power of beta rhythm decreases significantly both in resting state and task state, which is consistent with the change trend of its power. Consistent research results have also been widely reported [22, 23]. Beta rhythm is usually associated with the excited state of the brain (e.g., mood and mental activity). When the brain converts from resting state to task state, it needs to maintain a high concentration to complete the tasks, and its beta proportion rises from $15 \%$ to $28 \%$. According to the brain topography in the fifth column of Figs. 2 and 4, beta rhythm is mainly distributed in the temporal region, which is consistent with the results of the brain topography given by Jap et al. [23]. Based on the statistical results in Tables 3 and 5, the effect of beta rhythm on depicting mental fatigue in resting state is slightly better than that in task state.

\section{Conclusions}

In this study, we attempted to study the differences of mental fatigue on electrophysiological activity both in resting and task states at the same time. A group of mental arithmetic math problems was performed for mental fatigue induction. EEG data was collected before and after the tasks. Then five EEG rhythms (delta, theta, alpha1, alpha2, and beta) were calculated and discussed both in resting and task states. The results suggested the following conclusions: firstly, the task of mental arithmetic problems can successfully induce mental fatigue in the enrolled subjects; secondly, the relative power index of each EEG rhythm is more sensitive than the power index in response to mental fatigue, suggesting that relative power can be applied to estimate brain fatigue level; thirdly, the relative power of each EEG rhythm is better at assessing mental fatigue in resting state than in task state; finally, it is of great physiological significance to divide alpha frequency band into alpha1 band and alpha 2 band in fatigue related studies, and at the same time improve the statistical differences of sub-bands.

\section{Methods}

\section{Participants}

In this study, 20 right-handed and healthy male participants of engineering postgraduate students (age: $24.5 \pm 1.5$ years, BMI: $20.7 \pm 1.8 \mathrm{~kg} / \mathrm{m}^{2}$ ) were recruited. Each subject must have regular living habits and normal eyesight, and have no brain disorders. All participants were asked to follow the bellow requirements before the tests: (1) forbid to stay up late and drink alcohol and drugs within a week, (2) prohibit smoke, coffee and tea within $8 \mathrm{~h}$, and (3) wash the hair two hours ago. Every participant signed the informed consent, and Shandong University Ethics Committee approved our study. Detailed descriptions about the participants were introduced in the Ref. [40].

\section{EEG data recording and preprocessing}

A mental fatigue model was carried out to induce fatigue among all recruited participants: do two hundred different mental arithmetic problems (one number between sixty and ninety plus another number between sixty and ninety, and then multiplied by a number between six and nine) for one hundred minutes. All problems were strictly designed to be at appropriate difficulty level and can be finished within thirty-seconds according to preceding pretests. That is, all the participants can get high accuracies during the divided four tasks. The results of the accuracies were similar and had no statistic difference among these four tasks. What we concerned was the effects of tasks on the brain when the participants highly focused on the given mental arithmetic math tasks. Detailed descriptions about the experimental design were introduced in the Ref. [40].

As depicted in Fig. 6, these two hundred problems were evenly divided into four task segments, and 19-channel EEG data were recorded before and after these four task segments for both resting state and task state (2 minutes EEG data recordings for each state). Thus, five times EEG recordings named as T0, T1, T2, T3 and T4 were implemented in total. Then, 10 pieces of sequential 5-second EEG signals with no artifacts were selected by EEGLAB for each state (Only eighteen participants' EEG signals were further analyzed, because the other two were excluded due to their large head movements during EEG collections). EEG rhythms of delta $(2-4 \mathrm{~Hz})$,

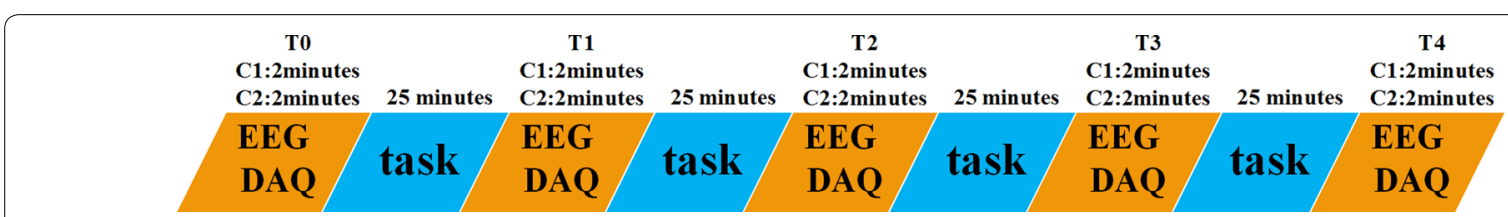

Fig. 6 EEG data acquisition (EEG DAQ) procedures. C1 means resting state and C2 means task state 
theta $(4-8 \mathrm{~Hz})$, alpha1 $(8-10 \mathrm{~Hz})$, alpha2 $(10-13 \mathrm{~Hz})$, and beta $(13-30 \mathrm{~Hz})$ were extracted by digital FFT filtering. Detailed descriptions and definitions about EEG data recordings and preprocessing were introduced in the Ref. [40].

\section{Computation of EEG indices}

In this study, the power and relative power of every EEG rhythms were explored. The frequency spectrum $X(f)$ of EEG signal $x(n)$ was obtained by means of FFT, and then the power spectrum $P_{x}(f)$ of EEG were gained with Eq. (1). The power $E(h)$ and relative power $R(h)$ were calculated through Eq. (2) and Eq. (3). Where, in Eq. (1), Eq. (2) and Eq. (3), $N$ is the number of EEG signal $x(n), h$ represents the EEG rhythms (such as delta, theta, alpha1, alpha2, beta), $f_{h}$ and $f_{l}$ are the upper and lower frequencies of $h$ rhythm respectively, $E_{\text {total }}$ is the total power of all EEG rhythms. Besides, all the calculated power spectrum are the average value of the selected 10-segment EEG signals for each condition. In order to study the differences of mental fatigue in different brain regions [23], we divided the whole brain region into five brain functional regions. As shown in Table 6, the nineteen electrodes are also divided into five groups. And EEG indices are also computed based on the five brain functional regions.

$$
\begin{aligned}
& P_{x}(f)=\frac{1}{N}|X(f)|^{2} . \\
& E(h)=\frac{1}{f_{h}-f_{l}} \int_{f_{l}}^{f_{h}} P_{x}(f) d f . \\
& R(h)=\frac{E(h)}{E_{\text {total }}} .
\end{aligned}
$$

\section{Statistical analysis}

One-way analysis of variance (ANOVA) was performed on the power and relative power of EEG bands to distinguish the statistically significant differences among the five periods. $P$-value, $F$-value and $\eta_{p}^{2}$ (partial eta squared) are given in the ANOVA results. In order to make the statistical results more convincing and reduce the risk of false positive, FDR (false discovery rate) correction was carried out for the $P$-values among different brain regions.

\section{Abbreviations}

EEG: Electroencephalogram; ANOVA: Analysis of variance; FDR: False discovery rate; EEG DAQ: EEG data acquisition.

\section{Acknowledgements}

Not applicable.

\section{Authors' contributions}

LG conception and design, experimental procedures, analysis and interpretation of the results, writing the article. JY and JW conception and design, analysis and interpretation of the results. XW and GZ perform and analyze experiments, and interpret results. HS and ZJ interpretation of the results, critical revision of the article. All authors proof-read the manuscript. All authors read and approved the final manuscript.

\section{Funding}

This work was partly supported by the National Natural Science Foundation of China (No. 51575497), Zhejiang Provincial Natural Science Foundation of China (No. LQ19E050011), and the Zhejiang Provincial Public Welfare Technology Application Research Foundation of China (No. LGF19G010005). These funders had no role in the design of the study and collection, analysis, and interpretation of data and in writing and publishing the manuscript.

\section{Availability of data and materials}

The datasets used and/or analyzed during the current study are available from the corresponding author on reasonable request.

\section{Ethics approval and consent to participate}

All procedures were approved by the Ethics Committee of Shandong University, and written informed consent was obtained from each participant.

\section{Consent to publish}

Not applicable.

\section{Competing interests}

The authors declare that they have no conflict of interest.

Received: 23 December 2019 Accepted: 30 April 2020

Published online: 12 May 2020

References

1. Boksem MAS, Tops M. Mental fatigue: costs and benefits. Brain Res Rev. 2008;59(1):125-39.

2. Jin SH, Lin P, Hallett M. Abnormal reorganization of functional cortical small-world networks in focal hand dystonia. PLoS ONE. 2011;6(12):12.

3. Ahn S, Nguyen T, Jang H, Kim JG, Jun SC. Exploring neuro-physiological correlates of drivers' mental fatigue caused by sleep deprivation using simultaneous EEG, ECG, and fNIRS data. Front Hum Neurosci. 2016;10:219.

4. Peng Q, Hua R, Lingyun G, Xiaobing Z, Tianshu Z, Yu T, Nitish T, Anastasios $B$, Jinsong $L$, Sun $Y$. Neural mechanisms of mental fatigue revisited: new insights from the brain connectome. Engineering. 2019. https://doi. org/10.1016/j.eng.2018.11.025.

5. Li G, Jiang Y, Jiao W, Xu W, Huang S, Gao Z, Zhang J, Wang C. The maximum eigenvalue of the brain functional network adjacency matrix: meaning and application in mental fatigue evaluation. Brain sci. 2020;10(2):92.

6. Ishii A, Tanaka M, Shigihara Y, Kanai E, Funakura M, Watanabe Y. Neural effects of prolonged mental fatigue: a magnetoencephalography study. Brain Res. 2013;1529:105-12.

7. Tanaka M, Ishii A, Watanabe Y. Neural effects of mental fatigue caused by continuous attention load: a magnetoencephalography study. Brain Res. 2014:1561:60-6.

8. Dimitrakopoulos GN, Kakkos I, Dai Z, Wang H, Sgarbas K, Thakor N, Bezerianos A, Sun Y. Functional connectivity analysis of mental fatigue reveals different network topological alterations between driving and vigilance tasks. IEEE Trans Neural Syst Rehabil Eng. 2018;26(4):740-9.

9. Sun Y, Lim J, Meng J, Kwok K, Thakor N, Bezerianos A. Discriminative analysis of brain functional connectivity patterns for mental fatigue classification. Ann Biomed Eng. 2014;42(10):2084-94.

10. Kar S, Bhagat M, Routray A. EEG signal analysis for the assessment and quantification of driver's fatigue. Transportation Res Part F Traffic Psychol Behav. 2010;13(5):297-306.

11. Li G, Li B, Wang G, Zhang J, Wang J. A new method for human mental fatigue detection with several EEG channels. J Med Biol Eng. 2017:37(2):240-7. 
12. $\mathrm{Hu} J, \mathrm{Min} J$. Automated detection of driver fatigue based on EEG signals using gradient boosting decision tree model. Cogn Neurodyn. 2018;12(4):431-40.

13. Lim J, Quevenco F-C, Kwok K. EEG alpha activity is associated with individual differences in post-break improvement. Neuroimage. 2013;76(1):81-9

14. Lawhern V, Kerick S, Robbins KA. Detecting alpha spindle events in EEG time series using adaptive autoregressive models. BMC Neurosci. 2013;14:101.

15. Huang J, Ulke C, Sander C, Jawinski P, Spada J, Hegerl U, Hensch T. Impact of brain arousal and time-on-task on autonomic nervous system activity in the wake-sleep transition. BMC Neurosci. 2018;19:11.

16. Tian S, Wang Y, Dong G, Pei W, Chen H. Mental fatigue estimation using eeg in a vigilance task and resting states. Conference proceedings: Annual International Conference of the IEEE Engineering in Medicine and Biology Society IEEE Engineering in Medicine and Biology Society Annual Conference. 2018;2018:1980-3.

17. Strijkstra AM, Beersma DGM, Drayer B, Halbesma N, Daan S. Subjective sleepiness correlates negatively with global alpha $(8-12 \mathrm{~Hz})$ and positively with central frontal theta $(4-8 \mathrm{~Hz})$ frequencies in the human resting awake electroencephalogram. Neurosci Lett. 2003;340(1):17-20.

18. Putilov AA, Donskaya OG. Alpha attenuation soon after closing the eyes as an objective indicator of sleepiness. Clin Exp Pharmacol Physiol. 2014;41(12):956-64

19. Chen J, Wang H, Hua C, Wang Q, Liu C. Graph analysis of functional brain network topology using minimum spanning tree in driver drowsiness. Cogn Neurodyn. 2018;12(6):569-81.

20. Li G, Li B, Jiang YH, Jiao WD, Lan H, Zhu CG. A new method for automatically modelling brain functional networks. Biomed Signal Process Control. 2018;45:70-9.

21. Sun Y, Lim J, Kwok K, Bezerianos A. Functional cortical connectivity analysis of mental fatigue unmasks hemispheric asymmetry and changes in small-world networks. Brain Cogn. 2014;85:220-30.

22. Eoh HJ, Chung MK, Kim S-H. Electroencephalographic study of drowsiness in simulated driving with sleep deprivation. Int J Ind Ergon. 2005;35(4):307-20.

23. Jap BT, Lal S, Fischer P, Bekiaris E. Using EEG spectral components to assess algorithms for detecting fatigue. Expert Syst Appl. 2009;36(2):2352-9.

24. Lal SKL, Craig A. Driver fatigue: electroencephalography and psychological assessment. Psychophysiology. 2002;39(3):313-21.

25. Åkerstedt T, Gillberg M. Subjective and objective sleepiness in the active individual. Int J Neurosci. 2009;52(1-2):29-37.

26. Lal SKL, Craig A. Reproducibility of the spectral components of the electroencephalogram during driver fatigue. Int J Psychophysiol. 2005;55(2):137-43.
27. Torsvall L, Akerstedt T. Sleepiness on the job: continuously measured EEG changes in train drivers. Electroencephalogr Clin Neurophysiol. 1987;66(6):502-11.

28. Hartman AL: Atlas of EEG Patterns: Lippincott Williams \& Wilkins; 2013.

29. Lal SKL, Craig A. A critical review of the psychophysiology of driver fatigue. Biol Psychol. 2001;55(3):173-94.

30. Craig A, Tran Y, Wijesuriya N, Hung N. Regional brain wave activity changes associated with fatigue. Psychophysiology. 2012;49(4):574-82.

31. Boksem MAS, Meijman TF, Lorist MM. Effects of mental fatigue on attention: an ERP study. Cogn Brain Res. 2005;25(1):107-16.

32. Klimesch W. EEG alpha and theta oscillations reflect cognitive and memory performance: a review and analysis. Brain Res Rev. 1999;29(2-3):169-95.

33. Klimesch W, Doppelmayr M, Pachinger T, Ripper B. Brain oscillations and human memory: EEG correlates in the upper alpha and theta band. Neurosci Lett. 1997;238(1-2):9-12.

34. Klimesch W, Doppelmayr M, Hanslmayr S. Upper alpha ERD and absolute power: their meaning for memory performance. Prog Brain Res. 2006;159(1):151-65

35. Sauseng P, Klimesch W, Schabus M, Doppelmayr M. Fronto-parietal EEG coherence in theta and upper alpha reflect central executive functions of working memory. Int J Psychophysiol. 2005;57(2):97-103.

36. Schack B, Klimesch W, Sauseng P. Phase synchronization between theta and upper alpha oscillations in a working memory task. Int J Psychophysiol. 2005;57(2):105-14.

37. Escolano C, Aguilar M, Minguez J, leee: EEG-based Upper Alpha Neurofeedback Training Improves Working Memory Performance. In: 33rd Annual International Conference of the IEEE Engineering-in-Medicineand-Biology-Society (EMBS): Aug 30-Sep 03 2011; Boston, MA: leee; 2011: 2327-2330.

38. Zoefel B, Huster RJ, Herrmann CS. Neurofeedback training of the upper alpha frequency band in EEG improves cognitive performance. Neurolmage. 2011;54(2):1427-31.

39. Klimesch W, Doppelmayr M, Pachinger T, Russegger H. Event-related desynchronization in the alpha band and the processing of semantic information. Cogn Brain Res. 1997;6(2):83-94.

40. Li G, Luo YD, Zhang ZR, Xu YT, Jiao WD, Jiang YH, Huang S, Wang CW. Effects of mental fatigue on small-world brain functional network organization. Neural Plasticity. 2019. https://doi.org/10.1155/2019/1716074.

\section{Publisher's Note}

Springer Nature remains neutral with regard to jurisdictional claims in published maps and institutional affiliations.
Ready to submit your research? Choose BMC and benefit from:

- fast, convenient online submission

- thorough peer review by experienced researchers in your field

- rapid publication on acceptance

- support for research data, including large and complex data types

- gold Open Access which fosters wider collaboration and increased citations

- maximum visibility for your research: over $100 \mathrm{M}$ website views per year

At BMC, research is always in progress.

Learn more biomedcentral.com/submissions 